\title{
Resolved Spectroscopy of the Narrow-Line Region in NGC 1068: Kinematics of the Ionized Gas ${ }^{1}$
}

\author{
D. Michael Crenshaw ${ }^{2} \&$ Steven B. Kraemer ${ }^{3}$ \\ Catholic University of America and Laboratory for Astronomy and Solar Physics, NASA's \\ Goddard Space Flight Center, Code 681, Greenbelt, MD 20771
}

Received __; accepted _

\footnotetext{
${ }^{1}$ Based on observations with the NASA/ESA Hubble Space Telescope, which is operated by the Association of Universities for Research in Astronomy, Inc., under NASA contract NAS5-26555.

${ }^{2}$ crenshaw@buckeye.gsfc.nasa.gov

${ }^{3}$ stiskraemer@yancey.gsfc.nasa.gov
} 


\begin{abstract}
We have determined the radial velocities of the [O III] emitting gas in the inner narrow-line region (NLR) of the Seyfert 2 galaxy NGC 1068, along a slit at position angle $202^{\circ}$, from STIS observations at a spatial resolution of $0^{\prime \prime} .1$ and a spectral resolving power of $\lambda / \Delta \lambda \approx 1000$. We use these data to investigate the kinematics of the NLR within $6^{\prime \prime}(\sim 430 \mathrm{pc})$ of the nucleus. The emission-line knots show evidence for radial acceleration, to a projected angular distance of $1^{\prime \prime} .7$ in most cases, followed by deceleration that approaches the systemic velocity at a projected distance of $\sim 4^{\prime \prime}$. We find that a simple kinematic model of biconical radial outflow can match the general trend of observed radial velocities. In this model, the emitting material is evacuated along the bicone axis, and the axis is inclined $5^{\circ}$ out of the plane of the sky. The acceleration of the emission-line clouds provides support for dynamical models that invoke radiation and/or wind pressure. We suggest that the deceleration of the clouds is due to their collision with a patchy and anistropically distributed ambient medium.
\end{abstract}

Subject headings: galaxies: individual (NGC 1068) - galaxies: Seyfert 


\section{Introduction}

The narrow-line region (NLR) in Seyfert galaxies is characterized by emission lines with widths on the order of $500 \mathrm{~km} \mathrm{~s}^{-1}$ (full-width at half-maximum, FWHM), which are attributed to motions of the ionized clouds of gas. Over the past couple of decades, ground-based studies attempted to determine the kinematics of the NLR, but a general consensus on the velocity flow pattern was not reached; cases were made for infall, rotation, parabolic orbits, outflow, etc. (e.g., Osterbrock and Mathews 1986, and references therein; DeRobertis \& Shaw 1990; Veilleux 1991; Moore \& Cohen 1994, 1996). Since the majority of the NLR flux comes from a region that subtends only a few arcseconds in most Seyferts (Schmitt \& Kinney 1996), these studies had to rely on spatially-integrated line profiles. Unfortunately, similar profile shapes and asymmetries can be generated from a wide variety of kinematic models (Capriotti, Foltz, \& Byard 1980, 1981), and hence the difficulty in determining the velocity fields from these data. By contrast, ground-based studies of the

extended narrow-line region (ENLR, at distances typically $\geq 500$ pc from the central source) can take advantage of of spatially-resolved spectra; these studies find that the ionized gas in the ENLR is undergoing normal galactic rotation (Unger et al. 1987), with evidence for an additional component of outward radial motion in some cases (Whittle et al. 1988). Despite the limited spatial resolution, recent ground-based studies suggest that the NLR of NGC 1068, the nearest bright Seyfert 2 galaxy, shows evidence for radial outflow (Cecil, Bland, \& Tully 1990; Arribas, Mediavilla, and García-Lorenzo 1996), which is a suggestion first offered by Walker (1968).

With the Hubble Space Telescope (HST) and the Space Telescope Imaging Spectrograph (STIS), we now have the ability to obtain spectra of the NLR at high spatial resolution. The importance of these observations is that we can probe the velocity field of the ionized gas close to the central continuum source, where the supermassive black hole presumably 
dominates the kinematics (due to its gravitational influence and/or the radiation, winds, and jets emanating from its vicinity). In this letter, we use STIS long-slit spectra of the Seyfert 2 galaxy NGC 1068 to determine the kinematics of the ionized gas in its NLR. In previous papers, we used these spectra to study the extended continuum emission (Crenshaw \& Kraemer 2000, hereafter Paper I) and the physical conditions in ionized gas near the continuum "hot spot" (Kraemer \& Crenshaw 2000, Paper II). We adopt a systemic redshift of $\mathrm{cz}=1148 \mathrm{~km} \mathrm{~s}^{-1}$ from H I observations of NGC 1068 (Brinks et al. 1997) and a distance of 14.4 Mpc (Bland-Hawthorne 1997), so that $0^{\prime \prime} .1$ corresponds to 7.2 pc.

\section{Observations and Results}

The observations and data reduction are described in detail in Paper I. Briefly, we obtained STIS low-dispersion spectra over the range 1150 - 10,270 $\AA$ at a spatial resolution of $0^{\prime \prime} .05-0^{\prime \prime} .1$ and a spectral revolving power of $\lambda / \Delta \lambda \approx 1000$ through a $0^{\prime \prime} .1$ slit at a position angle of $202^{\circ}$. The slit position intercepts the brightest clouds in the inner NLR, as shown in Paper I. Here we concentrate on the brightest emission line, [O III] $\lambda 5007$, to trace the kinematics as far as possible away from the nucleus.

Figure 1 presents an enlarged view of the STIS spectrum, in the region around the $\mathrm{H} \beta$ and [O III] $\lambda \lambda 4959,5007$ lines, which show a considerable amount of spatial and velocity structure. The most striking aspect of these lines is that they split into two velocity components both above (SW of) and below (NE of) the spectrum of the continuum hot spot (the horizontal streak). The brightest emission-line clouds show a definite trend of increasing radial velocity with distance from the hot spot, out to an angular distance of about $\pm 1^{\prime \prime} .7$. Most of the fainter clouds follow this trend, and show an overall decrease in radial velocity further out, until they approach the systemic velocity. There are a few exceptions to these trends, which we will discuss below. 
We determined radial velocities from the [O III $\lambda$ 25007 emission at each pixel location along the slit (at a spacing of $0^{\prime \prime} .05$ ). At each location, we fit the emission with a local continuum and a Gaussian for each clearly identifiable peak, resulting in 1 or 2 kinematic components. A number of the observed components, particularly near the hot spot, are asymmetric and/or very broad, and we suspect that these may split into multiple components at higher spectral resolution. In Figure 2, we plot the heliocentric radial velocities, widths (FWHM, corrected for the instrumental profile), and fluxes as a function of distance from the peak of the hot spot in our slit (which is $0^{\prime \prime} .14$ north of the hot spot's centroid in HST optical continuum images, see Paper I).

Most of the radial velocities in Figure 2 follow well-defined curves; the local peaks in the radial velocity curves can be attributed in many cases to bulk motion of the emission-line knots seen in the bottom plot. The pattern of increasing velocity out to $\sim 1^{\prime \prime} .7$ is evident, except for the blueshifted knots on the SW side, which reach a smaller maximum velocity closer in $\left(\sim 1^{\prime \prime}\right)$. The decrease in radial velocity at greater distances is also clear, except that the redshifted knots in the SW abruptly terminate at about $2^{\prime \prime} .0$. There are two knots of emission NE of the hot spot that do not conform to this pattern at all; one is highly

blueshifted $\left(-1400 \mathrm{~km} \mathrm{~s}^{-1}\right)$ and the other is highly redshifted $\left(+1000 \mathrm{~km} \mathrm{~s}^{-1}\right)$ with respect to the systemic velocity. The middle plot in Figure 2 shows the large widths of the lines, which tend to decrease with distance, particularly in the SW.

\section{A Kinematic Model: Biconical Outflow}

HST images indicate a biconical geometry for the NLR in most Seyfert 2 galaxies (Schmitt \& Kinney 1996), and for NGC 1068 in particular (Evans et al. 1991). The radial velocity curves in Figure 2 suggest a velocity field in which the emission-line knots accelerate out from the inner nucleus, reach a terminal velocity, and then decelerate. Thus, 
we favor a kinematic model of biconical outflow away from the nucleus. Similar amplitudes of the blueshifted and redshifted curves on the NE side indicate that the axis of the bicone is close to the plane of the sky, and the lack of low radial velocities where the curves peak (around $\pm 1^{\prime \prime} .7$ ) suggests that the bicone is evacuated along its axis.

We have generated a kinematic model of radial outflow in a bicone that is hollow along its axis. We constrain our model to be consistent with the observed morphology: Evans et al. (1991) find that the NLR in NGC 1068 can be described by a bicone with a projected half-opening angle of $\sim 35^{\circ}$ and a position angle of the bicone axis on the sky of $15^{\circ}$. For simplicity, we assume that the two cones have identical properties (geometry, velocity law, etc.), a filling factor of one within the defined geometry, and no absorption of [O III] photons (e.g., by dust). The parameters that are allowed to vary in our code are the extent of each cone along its axis $\left(\mathrm{z}_{\max }\right)$, its minimum and maximum half-opening angles $\left(\theta_{\text {inner }}\right.$ and $\left.\theta_{\text {outer }}\right)$, the inclination of its axis out of the plane of the sky $\left(\mathrm{i}_{\text {axis }}\right)$, and the velocity law as a function of distance from the nucleus. For the latter, we will show that constant acceleration to a maximum velocity $\left(\mathrm{v}_{\max }\right)$ at a turnover radius $\left(\mathrm{r}_{t}\right)$, followed by a constant deceleration to zero velocity at the greatest extent of the cone $\left(=\mathrm{z}_{\max } / \cos \theta_{\text {outer }}\right)$, provide a reasonable match to the observations.

Our code generates a two-dimensional velocity map and samples this map with a slit that matches the position, orientation, and width of our observational slit (Paper I); in this case, our slit is placed $0^{\prime \prime} .14$ north of the hot spot centroid and rotated $7^{\circ}$ with respect to the projected bicone axis. We then compare the simulated and observed radial velocity curves, and adjust the model parameters until a good match is obtained. Since the observed radial velocity curves have significant intrinsic scatter, we do not fine-tune the models (e.g., by choosing different turnover locations and maximum velocities for each side), but settle for an illustrative model that matches the overall trend. The parameters for our final model 
are given in Table 1.

Figure 3 shows the envelope of radial velocities from the model, compared to the observed radial velocities; the width of the envelope is determined by the range in half-opening angle $\left(\theta_{\text {inner }}\right.$ to $\left.\theta_{\text {outer }}\right)$, and the relative amplitudes of blue and redshifted curves are determined by the inclination angle $\left(\mathrm{i}_{\text {axis }}\right)$. In three quadrants of the plot in Figure 3, the overall trend of observed radial velocities is well matched by the model. ff For the blueshifted emission on the SW side, the radial velocities reach a smaller maximum closer in $\left(\right.$ at $\sim 1^{\prime \prime}$ ), and show a slight trend towards deceleration further out. As mentioned previously, there are two emission-line knots at very high velocities that do not fit this model at all. The presence of additional points outside of the envelope indicates that there may be a few emission-line knots close to the axis or knots that undergo slightly different accelerations or decelerations. Thus, although this model is simplistic, we adopt it as a tool for interpreting the velocity field, and discuss ways in which the descrepancies can be accommodated.

\section{Discussion}

\subsection{Comparison with Ground-based Observations}

Cecil et al. (1990) provide the most comprehensive set of velocity maps for the NLR of NGC 1068, based on Fabry-Perot observations of the [N II] lines at $\sim 1^{\prime \prime}$ spatial and 140 $\mathrm{km} \mathrm{s}^{-1}$ spectral resolutions; these authors conclude that their data can be explained by

\footnotetext{
${ }^{4}$ Note that we have assumed that the origin of the outflow is the continuum hot spot; if the origin is at the S1 radio component (Gallimore, Baum, \& O'Dea 1996), which is 0".17 S of the hot spot (Capetti, Macchetto, \& Lattanzi 1997), we find that only minor adjustments are needed in the half-opening angles and turnover radius to match the oberved trend.
} 
cylindrical or biconical outflow. Given the limited spatial resolution, their observations are in agreement with ours and are consistent with our model. Their line profiles along the STIS slit position show a single broad component at the location of the hot spot and double-peaked profiles, separated by $\sim 1000 \mathrm{~km} \mathrm{~s}^{-1}$, at a distance $\sim 2^{\prime \prime}$ from the hot spot, in agreement with our observations (Figure 3). Outside of the STIS slit position, their profiles show the same double-peaked structure, with the largest velocity separation at $\sim 2^{\prime \prime}$ on either side of the hot spot, which is consistent with our model prediction that the highest blueshifts and redshifts should occur at this distance. The higher spatial resolution of the STIS observations allows us to see the acceleration of clouds outward from the nucleus followed by a clear deceleration of the clouds. We have not applied our model to ground-based observations at distances $>6^{\prime \prime}$, where galactic rotation begins to play a much larger role in the kinematics (Baldwin, Whittle, \& Wilson 1987).

\subsection{Comparison with Other Models}

Two other kinematic models of the NLR have been proposed on the basis of spectra at high spatial resolution, which were obtained with HST's Faint Object Camera (FOC). Axon et al. (1998) suggest a model for NGC 1068 in which the gas expands outward from the radio jet (which is nearly coincident with the axis of the ionization cone). Winge et al. (1999) propose a rotating disk model for the NLR in the Seyfert 1 galaxy NGC 4151. To

test the Axon et al. (1998) suggestion for NGC 1068, we generated a model with the same parameters as in Table 1, except that the velocity vectors are directed perpendicular to the radio axis. In this case, we find that the envelope of radial velocities is nearly the same as in Figure 3, due to the small inclination angle. However, we have two concerns about this model. First, the observed radial velocities follow a well-organized flow pattern, and we can discern no correlation with the clumpy radio structure in the NLR (Gallimore et al. 1996). 
Second, this model cannot explain the kinematics of the NLR in the Seyfert 1 galaxy NGC 4151, where the gas is blueshifted in one cone and redshifted in the other (Winge et al. 1999; Nelson et. al. 2000, and references therein), whereas motions perpendicular to the axis produce equal blueshifts and redshifts in each cone, regardless of the inclination of the axis. We note that our radial outflow model for NGC 1068 provides a slightly better fit than the Axon et al. model, because a small tilt of the axis $\left(5^{\circ}\right)$ can match the slightly different amplitudes of the observed blueshifted and redshifted curves. Furthermore, by tilting the cone axis towards the line of sight, this model can explain the observed radial velocities in NGC 4151 (see Crenshaw et al. 2000).

Winge et al.'s (1999) rotating disk model for NGC 4151 is only used to match their low velocity component (within $300 \mathrm{~km} \mathrm{~s}^{-1}$ of systemic). Even so, they require an extended (and otherwise undetected) distribution of matter within $0^{\prime \prime} .1$ (64 pc) of the nucleus with a mass on the order of $10^{9} \mathrm{M} \odot$, in addition to a central point-source mass of $10^{7} \mathrm{M} \odot$. A rotation model can be ruled out for NGC 1068, because high redshifts and blueshifts are seen on either side of the nucleus. Other gravitational models can also be ruled out as the principal source of the velocities in NGC 1068, because the required mass is prohibitive. At the peaks of the velocity curves (ignoring the two knots with very high velocities), the projected distance from the nucleus is $\sim 120 \mathrm{pc}$, the projected velocity is $\sim 850 \mathrm{~km}$ $\mathrm{s}^{-1}$, and the required mass is $\geq 10^{10} \mathrm{M} \odot$ (a lower limit because of projection effects). By comparison, the dynamical mass from stars within a radius of $\sim 1^{\prime \prime}$ from the nucleus of NGC 1068 is only $6 \times 10^{8} \mathrm{M} \odot$ (Thatte et al. 1997). Thus, radial outflow provides the simplest and best explanation of the observed velocities in these two Seyfert galaxies. 


\subsection{Implications of our Model}

Our kinematic model assumes constant acceleration of clouds in the inner NLR $(<140$ pc), and constant deceleration further out; this assumption provides a reasonable match to the observations, although more complicated velocity laws as a function of distance are possible, given the intrinsic scatter in the observed points. Nevertheless, these results favor dynamical models that invoke radiation and/or wind pressure to drive clouds out from the nucleus. The deceleration of clouds further is not primarily due to gravity, for the reasons given above: an unreasonably high mass $\left(\sim 10^{10} \mathrm{M} \odot\right)$ is required to slow the clouds down. The simplest explanation for the deceleration is that the clouds experience a drag force, presumably due to interaction with ambient material at $\sim 140$ pc from the nucleus. A possible explanation for the blueshifted clouds on the SW side is that they run into ambient material that is closer to the nucleus $(\sim 80 \mathrm{pc})$. In this picture, the two high velocity clumps in Figure 3 represent clouds that have not experienced a drag force in the direction they are traveling, which suggests that there are holes in the surrounding medium. We note that gravity may eventually play a role in the kinematics. In our model, the axis of the outflow is inclined by $\sim 45^{\circ}$ with respect to the galactic disk (inclination $=40^{\circ}$, major axis at position angle $106^{\circ}$, see Bland-Hawthorne et al. 1997), and as the NLR clouds slow down, they may be pulled back to the disk, possibly joining the existing ENLR gas.

In conclusion, we find that a biconical outflow model, with evidence for acceleration close to the nucleus and deceleration further out, provides a reasonable explanation for the radial velocities in our long-slit spectrum of NGC 1068. STIS long-slit observations of NGC 1068 at higher spectral resolution and at different slit positions will be helpful in resolving velocity components and mapping out the two-dimensional velocity field. STIS observations of other Seyferts will help test the utility of this model. 
This work was supported by NASA Guaranteed Time Observer funding to the STIS Science Team under NASA grant NAG 5-4103. 


\section{REFERENCES}

Arribas, S., Mediavilla, E., \& García-Lorenzo, B. 1996, ApJ, 463, 509

Axon, D.J., Marconi, A., Capetti, a., Macchetto, F.d., Shreier, E., \& Robinson, A. 1998, ApJ, 496, L75

Baldwin, J.A., Wilson, A.S., \& Whittle, M. 1987, ApJ, 319, 84

Bland-Hawthorn, J., Gallimore, J.F., Tacconi, L.J., Brinks, E., Baum, S.A., Antonucci, R.R.J., \& Cecil, G.N. 1997, Ap\&SS, 248, 9

Brinks, E., Skillman, E.D., Terlevich, R.J., \& Terlevich, E.T. 1997, Ap\&SS, 248, 23

Capetti, A., Macchetto, F.D., and Lattanzi, M.G. 1997, Ap\&SS, 248, 245

Capriotti, E., Foltz, C., \& Byard, P. 1980, ApJ, 241, 903

Capriotti, E., Foltz, C., \& Byard, P. 1981, ApJ, 245, 396

Cecil, G., Bland, J., \& Tully, R.B. 1990, ApJ, 355, 70

Crenshaw, D.M. \& Kraemer, S.B. 2000, ApJ, in press (Paper I)

Crenshaw, D.M., et al. 2000, in preparation.

De Robertis, M.M. \& Shaw, R.A. 1990, ApJ, 348, 421

Evans, I.N., Ford, H.C., Kinney, A.L., Antonucci, R.R.J., Armus, L., \& Caganoff, S. 1991, ApJ, 369, L27

Kraemer, S.B. \& Crenshaw, D.M. 2000, ApJ, in press (Paper II)

Gallimore, J.F., Baum, S.A., \& O'Dea, C.P. 1996, ApJ, 464, 198

Moore, D. \& Cohen, R.D. 1994, ApJ, 433, 602

Moore, D. \& Cohen, R.D. 1996, ApJ, 470, 301

Nelson, C.H., Weistrop, D., Hutchings, J.B., Crenshaw, D.M., Gull, T.R., Kaiser, M.E., Kraemer, S.B., \& Lindler, D. 2000, ApJ, in press 
Osterbrock, D.E. \& Mathews, W.G. 1986, ARA\&A, 24, 171

Schmitt, H.R. \& Kinney, A.L. 1996, ApJ, 463, 498

Thatte, N., et al. 1997, Ap\&SS, 248, 225

Unger, S.W., Pedlar, A., Axon, D.J., Whittle, M., Meurs, E.J.A., \& Ward, M.J. 1987, MNRAS, 228, 671

Veilleux, S. 1991, ApJS, 75, 383

Walker, M.F. 1968, ApJ, 151, 71

Whittle, M., Pedlar, A., Muers, E.J.A., Unger, S.W., Axon, D.J., \& Ward, M.J. 1988, ApJ, 326,125

Winge, C., Axon, D.J., Macchetto, F.D., Capetti, A., \& Marconi, A. 1999, ApJ, 519, 134 
Fig. 1. - Expanded view of the STIS G430L spectral image of NGC 1068, showing the H $\beta$ and [O III] $\lambda \lambda 4959,5007$ lines (wavelength increases to the right). The slit position angle is $202^{\circ}$; NE is below and $\mathrm{SW}$ is above the spectrum of the hot spot (the bright continuum spectrum). (The bright column to the left of the [O III] $\lambda 4959$ line is a bad CCD column.) The spatial extent of this figure is $10^{\prime \prime}$.

Fig. 2.- Radial velocity, width (FWHM, corrected for instrumental width), and log flux (ergs s${ }^{-1} \mathrm{~cm}^{-2}$ per cross-dispersion pixel) of the [O III] $\lambda 5007$ emission along the slit, as a function of angular distance from the peak of the hot spot spectrum. Negative positions are towards the NE. In the bottom two plots, filled and open squares represent blueshifted and redshifted emission, respectively (relative to systemic).

Fig. 3.- Comparison of observed and model radial velocities. Errors in the observed radial velocities are smaller than the symbols, except as noted. The shaded region is the envelope of predicted radial velocities from the kinematic model described in the text. 
Table 1. Kinematic Model of NGC 1068

\begin{tabular}{ll}
\hline \hline Parameter & \multicolumn{1}{c}{ Value } \\
\hline & \\
$\mathrm{z}_{\text {max }}$ & $306 \mathrm{pc}$ \\
$\theta_{\text {inner }}$ & $26^{\circ}$ \\
$\theta_{\text {outer }}$ & $40^{\circ}$ \\
$\mathrm{i}_{\text {axis }}$ & $5^{\circ}(\mathrm{NE}$ is closer $)$ \\
$\mathrm{v}_{\text {max }}$ & $1300 \mathrm{~km} \mathrm{~s}^{-1}$ \\
$\mathrm{r}_{t}$ & $137 \mathrm{pc}$ \\
\hline
\end{tabular}




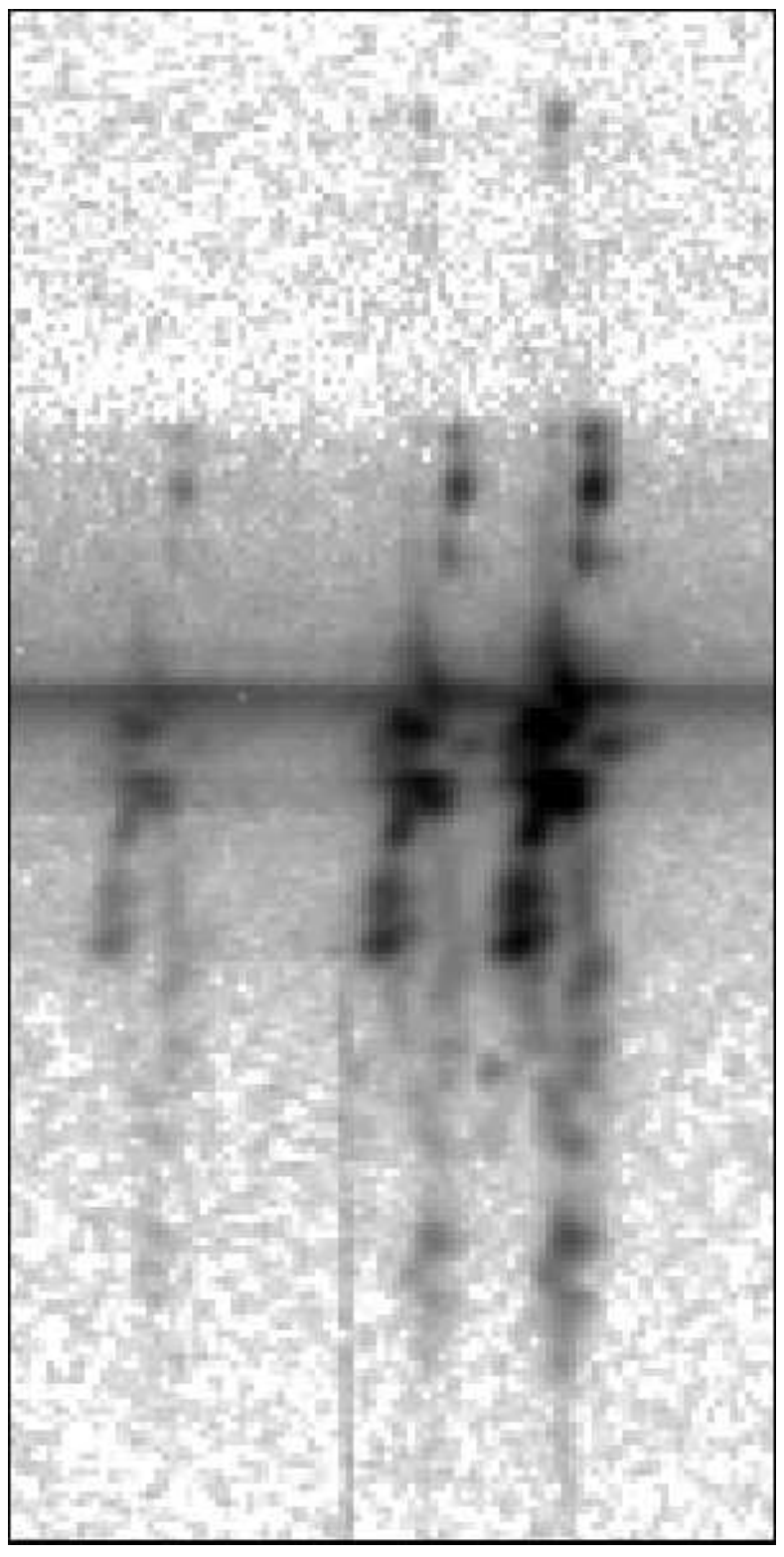

Fig. 1. 


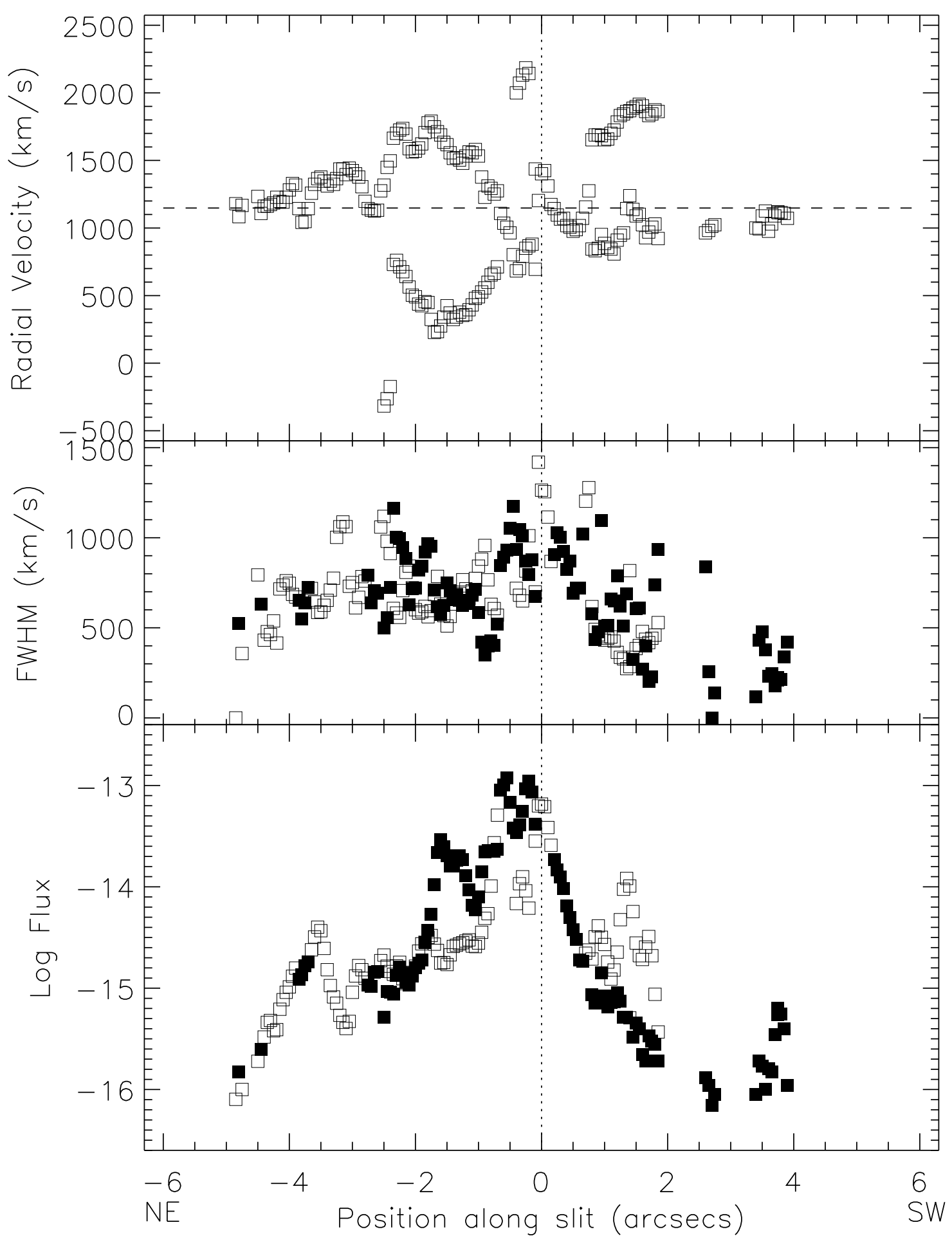

Fig. 2. 


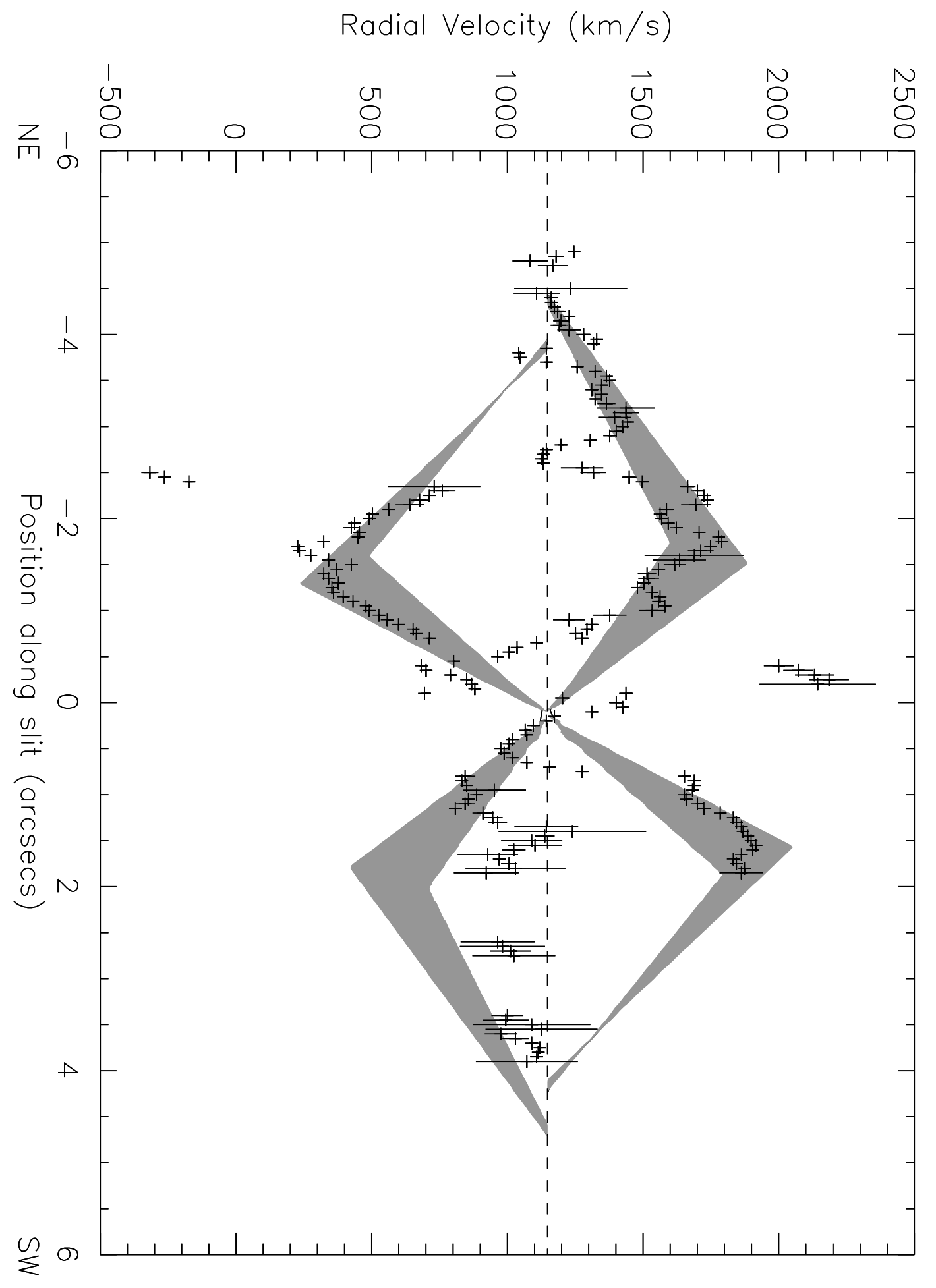

Fig. 3. 